\title{
Amended Data on Arginine Utilization by Spiroplasma Species
}

\author{
K. J. HACKETT, ${ }^{1 *}$ E. A. CLARK,${ }^{1}$ R. F. WHITCOMB,${ }^{1}$ M. CAMP ${ }^{2}$ AND J. G. TULLY ${ }^{3}$ \\ Insect Biocontrol Laboratory ${ }^{1}$ and Biometrical Consulting Service, ${ }^{2}$ U.S. Department of Agriculture, Beltsville, \\ Maryland 20705, and Mycoplasma Section, Laboratory of Molecular Microbiology, National Institute of Allergy and \\ Infectious Diseases, Frederick Cancer Research Facility, Frederick, Maryland $21701^{3}$

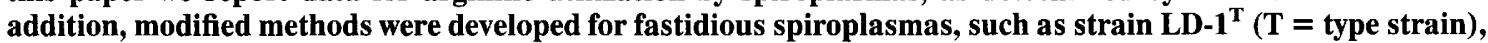
the Colorado potato beetle spiroplasma. Twenty-one spiroplasma strains representing 13 groups or subgroups and eight ungrouped spiroplasmas (seven of which represent putative groups) were studied. The arginine reactions of eight strains were the same as the reactions reported previously, but previously reported positive tests for spiroplasma subgroups I-5 and I-6 (Spiroplasma insolitum) could not be repeated, and the data for the latter taxa are corrected. Although other workers have reported that addition of carbohydrate to media may be necessary for the utilization of arginine, the presence of glucose tended to obscure arginine hydrolysis in our studies.
\end{abstract} \\ Hydrolysis of arginine is a classical diagnostic test for species in the mollicute order Entomoplasmatales. In \\ this paper we report data for arginine utilization by spiroplasmas, as determined by standard methods. In
}

Hydrolysis of arginine is an important diagnostic test for species of the mollicute order Entomoplasmatales (16). The arginine hydrolysis $(\mathrm{AH})$ test, as described by Barile (2), measures the production of ammonia released from arginine via the arginine dihydrolase pathway (3). Several lines of evidence have established that this pathway occurs in spiroplasmas; this evidence includes the presence of arginine deiminase activity and the accumulation of ornithine and citrulline during arginine degradation $(7,14,15)$, the results of labeled arginine experiments which show that label appears in ornithine (13), and the presence of an arginine-ornithine membrane exchange system in the AH-positive organism Spiroplasma melliferum (13).

In mollicutes, the arginine dihydrolase pathway may be linked to energy production (e.g., small amounts of energy [1 mol of ATP per mol of arginine] [3] are generated) or may serve as a source of carbon or nitrogen $(3,7)$. This pathway also functions in bacteria as a protection against increased acidity or osmolarity (9).

Although Mesoplasma and Entomoplasma species do not hydrolyze arginine, nine Spiroplasma groups and all eight subgroups of spiroplasma group I have been reported to be $\mathrm{AH}$ positive (18). However, we repeated tests for the group I spiroplasmas and were unable to confirm some previous results. In this paper, we amend previously reported data for two group I spiroplasmas (strains LB-12 [subgroup I-5] and $\mathrm{M} 55^{\mathrm{T}}$ $[\mathrm{T}=$ type strain] [subgroup I-6] [Spiroplasma insolitum]) and describe the reactions of 11 new strains, 7 of which are candidates for group status.

\section{MATERIALS AND METHODS}

Spiroplasma strains. Techniques for isolating spiroplasmas from flowers and insects have been described previously (20). The following spiroplasma strains, which are representative of or belong to previously described groups, were examined in this study: $277 \mathrm{~F}$ (passage 31 ; subgroup I-4), from a rabbit tick in Montana (21); LB-12 (passage 37; subgroup I-5), from a green leaf bug in Taiwan

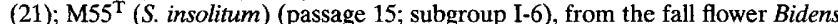
sp. (Asteraceae) in Maryland (21); N525 (passage 14; subgroup I-7), from a

* Corresponding author. Mailing address: Insect Biocontrol Laboratory, ARS, USDA, Room 214, Building 011A, BARC-W, Beltsville, MD 20705. Phone: (301) 504-6325. Fax: (301) 504-5104. Electronic mail address: khackett@asrr.arsusda.gov.
Cocos plant in Jamaica (21); $\mathrm{P} 40^{\mathrm{T}}$ (Spiroplasma phoeniceum) (passage 106; subgroup I-8), from a periwinkle plant in Syria (21); MO-1 ${ }^{\mathrm{T}}$ (Spiroplasma monobiae) (passage 77; group VII), from a Monobia quadridens wasp in Maryland (21); TAAS-1 (passage 14; subgroup VIII-3), from the gut of the horsefly Tabanus atratus in Texas (17); EC-1 ${ }^{\mathrm{T}}$ (Spiroplasma corruscae) (passage $>6$; group XIV), from the firefly Ellchynia comusca in Maryland (21); TAAS-2 (passage 14; closely related to TN-1; group XVIII), from the horsefly Tabanus atratus in Georgia (17); LD-1 ${ }^{\mathrm{T}}$ (Spiroplasma leptinotarsae) (passage 290; group XX), from the Colorado potato beetle Leptinotarsa decemlineata in Maryland (17); CT-1 ${ }^{\mathrm{T}}$ (Spiroplasma taiwanense) (passage 44; group XXII), from the mosquito Culex tritae niorynchus in Taiwan (21); TASS-1 (passage 5; related to TG-1; group XXIII) from the horsefly Tabanus sulcifrons in Georgia (21); and CUAS-1 ${ }^{\mathrm{T}}$ (Spiroplasma diminutum) (passage 22; group XXV), from the mosquito Culex annulus in Taiwan (21). The following seven ungrouped strains, which are representative of new putative groups, were also examined: BIUS-1 (passage 57), from a Bidens sp. flower surface in Maryland (17); HYOS-1 (passage 16), from the gut of the horsefly Hybomitra opaca in Montana (17)]; PALS-1, (passage 10), from the gut of the dragonfly Pachydiplax longipennis (Odonata: Libellulidae) in Maryland (17); PLHS-1 (passage 20), from a female common scorpionfly (Planorpa helena [Mecoptera: Planorpidae]) in West Virginia (17); TABS-2 (passage 11), from the gut of the horsefly Tabanus abactor in Oklahoma (17); TALS-2 (passage 38), from the gut of the horsefly Tabanus lineola in Georgia (17); TAUS-1 (passage 18), from the hemolymph of a horsefly in the Tabanus abdominalis-limbatinevris species complex in Maryland (17); and TABS-1 (passage 14; grouped with TAUS-1), from a horsefly in Texas (17).

Culture media and cultivation techniques. The serum fraction (SF) medium used in most tests was Townsend's BSM medium (15), with the following modifications: SF medium contained 1.5\% (wt/vol) BBL Mycoplasma Broth Base instead of the $2.1 \%$ BBL Mycoplasma Broth Base in BSM medium, 2.5\% (wt/ vol) $\mathrm{Gibco} / \mathrm{BRL}$ yeast extract instead of the $0.5 \% \mathrm{Gibco} / \mathrm{BRL}$ yeast extract in BSM medium, sorbitol (instead of $\mathrm{NaCl}$ ) for osmotic adjustment to $500 \mathrm{mOsM}$, and $2.0 \%$ (vol/vol) Difco Bacto PPLO serum fraction (instead of $15 \%$ horse serum). To eliminate contaminants, filtration with $0.22-\mu \mathrm{m}$-pore-size filters (Millex) and penicillin $(1,000 \mathrm{U} / \mathrm{ml})$ were used instead of thallous acetate. SF medium supplemented with glucose (SFG medium) contained $0.5 \%$ (wt/vol) glucose (instead of $0.1 \%$ glucose) and no fructose (instead of $0.1 \%$ fructose). SF medium supplemented with arginine (SFA medium) contained $0.42 \%$ (wt/vol) L-arginineHCl. SFGA medium contained both glucose and arginine at the concentrations indicated above. For additional experiments with strain $\mathrm{M} 55^{\mathrm{T}}$, a second modified Townsend medium (MTBSM medium) was used. MTBSM medium consisted of SFA medium (containing $0.42 \%$ arginine) plus $0.1 \%$ glucose and $0.1 \%$ fructose

Since spiroplasmas typically hydrolyze arginine at low $\mathrm{pH}$ values, the possible stimulatory effect of low $\mathrm{pH}$ values was tested by adjusting $\mathrm{SF}$ medium variants to $\mathrm{pH} 6.0$ to 6.3 and 6.8 to 7.0 and using the resulting preparations to tes subgroup I-6 strain M55 ${ }^{\mathrm{T}}$, which previously had been reported to be $\mathrm{AH}$ positive (18) but was AH negative in SFA medium.

To test arginine utilization by group XX strain LD- $1^{\mathrm{T}}$, SF-based media were unsuitable. Instead, DCCM medium $(4,5)$ containing $10 \%$ fetal bovine serum, $0.13 \%$ sugar, and $0.12 \%$ arginine (DBF medium) was modified so that it contained $0.33 \%$ arginine; the resulting medium was designated DBFA medium (6). Other medium variants contained an additional $0.5 \%$ glucose (DBFG medium) or additional glucose and arginine (DBFGA medium).

Arginine tests. The AH test procedure used was the procedure described by Barile (2), which was modified from the procedure of Aluotto et al. (1), with the 
following additional modifications. All strains except LD- $1^{\mathrm{T}}$ were first adapted from M1D medium (5) to FBSI medium (1.5\% BBL Mycoplasma Broth Base, $2.5 \% \mathrm{Gibco} / \mathrm{BRL}$ yeast extract, $20 \%$ heat-fixed [5] Gibco/BRL fetal bovine serum, $0.21 \%$ arginine, $0.5 \%$ glucose, $0.02 \mu \mathrm{g}$ of phenol red per ml; $\mathrm{pH} \mathrm{7.7;}$ osmolarity adjusted to $500 \mathrm{mOsM}$ with sorbitol). After the organisms had been passaged three times in FBSI medium, they were inoculated into the four test media (SF, SFG, SFA, and SFGA media). Only those cultures that exhibited good helical morphology were selected. A high-dilution passage of each mid- to late-log-phase culture ( $3 \mu \mathrm{l}$ of inoculum was added to $3.0 \mathrm{ml}$ of medium) was performed to minimize changes in the initial $\mathrm{pH}$ and the glucose concentration. All cultures and an uninoculated control were incubated at $32^{\circ} \mathrm{C}$ and examined daily for color change. For most trials, SFGA medium was used to make the set of phenol red-containing colorimetric standards; the $\mathrm{pH}$ values of these standards ranged from 6.0 to 8.4 in increments of $0.1 \mathrm{pH}$ unit. For tests performed with strain LD-1 ${ }^{\mathrm{T}}, 0.002 \%$ bromcresol purple was added to test media as a $\mathrm{pH}$ indicator. A DCCM growth medium color series (pH 5.8 to 7.4, with increments of $0.2 \mathrm{pH}$ unit) was used as the standard. A Corning model $240 \mathrm{pH}$ meter (sensitivity, $0.01 \mathrm{pH}$ unit) was used to verify some measurements.

In an additional test of strain M55 ${ }^{\mathrm{T}}$, the spiroplasma was adapted to SFGA 35 medium, which contained SFGA medium and SFA medium at a ratio of 1:35. Strain M55 $^{\mathrm{T}}$ was tested in SFGA35 medium, in MTBSM medium, and in a hybrid medium (TWH medium). TWH medium contained $2.1 \%$ BBL Mycoplasma Broth Base, $0.42 \%$ arginine, $0.014 \%$ glucose, $0.5 \%$ yeast extract, and $2 \%$ serum fraction. The previously reported AH by strains $277 \mathrm{~F}$ and N525 was confirmed by using a mixture of SFGA medium and SFA medium (1:2). Strain LB-12 was grown for several passages in $\mathrm{pH} 7.3$ SFGA medium-SFA medium mixtures (1:1 to $1: 10)$ and in SFA medium, and the $\mathrm{pH}$ was determined in each medium.

Statistical analysis. $\mathrm{pH}$ values for each day were plotted by strain for the four growth media used (SF, SFA, SFG, and SFGA media). Smooth curves were fitted to the points by using PROC GPLOT (12). The smoothing method which was used was a cubic spline that minimizes a linear combination of the sum of squares of the residuals of fit and the integral of the square of the second derivative (10); a smoothness value of 0 gives straight lines between the data points and a value of 99 gives a straight line that ignores the data points. The arginine graphs were plotted with a smoothness value of 43 .

\section{RESULTS}

Arginine utilization by spiroplasma strains. With the arginine concentrations used $(0.21,0.33$, and $0.42 \%)$, arginine utilization by AH-positive strains was evident in SFA medium but not in SF, SFG, or SFGA medium. The variants of SFA medium typically became acidified to $\mathrm{pH} \leq 6.0$. The acidification curves for AH-negative organisms in SF, SFG, and SFGA media were similar to the acidification curves for strain TAUS-1 in SFA medium (Fig. 1). AH-positive strains sometimes equilibrated at a higher minimal $\mathrm{pH}(\mathrm{pH} 6.4$ to 6.7$)$ in the stationary phase in SF medium than $\mathrm{AH}$-negative strains did ( $\mathrm{pH} \leq 6.0$ to 6.4 ). Some arginine utilizers had slightly higher SFG medium (strain P40, $\mathrm{pH}$ 6.4) and SFGA medium (strain P40, pH 6.4; strain PALS-1, pH 6.4; strain PLHS-1, pH 6.4) $\mathrm{pH}$ values than other strains $(\mathrm{pH} \leq 6.0)$. Strain $\mathrm{LD}-1^{\mathrm{T}}$ was exceptional in that it exhibited an increase in the $\mathrm{pH}$ (to $\mathrm{pH}$ $\geq 7.4$ ) in a medium containing glucose and arginine (DBFGA medium); however, in this case, DCCM medium had been used as the basal medium.

Strains 277F (subgroup I-4) and N525 (subgroup I-7) were previously reported to be AH positive (18). This was confirmed in the current study (data not shown). Also as previously reported (18), strain $\mathrm{P} 40^{\mathrm{T}}$ (subgroup I-8) was $\mathrm{AH}$ positive (Table 1).

In contrast to a previous report (18), we were not able to demonstrate AH by strains LB-12 (subgroup I-5) and M55 (subgroup I-6) (Table 1; Fig. 1). Strain LB-12 did not noticeably catabolize arginine in various SFGA medium-SFA medium mixtures at ratios of 1:1 to $1: 10$ or in SFA medium alone. When media with an initial $\mathrm{pH}$ of 7.3 were used, strain LB-12 lowered the $\mathrm{pH}$ only slightly or not at all, while achieving high titers (more than $10^{9}$ cells per $\mathrm{ml}$ ). Somewhat similarly, the $\mathrm{pH}$ values of $\mathrm{M} 55^{\mathrm{T}}$ cultures (in the trial in which a color standard was used) declined from $\mathrm{pH} 7.7$ to 6.4 in SFA medium. This stationary-phase equilibrium value was higher than the values
TABLE 1. Spiroplasma AH test results

\begin{tabular}{|c|c|c|c|c|c|c|}
\hline \multicolumn{2}{|c|}{ Spiroplasma } & \multirow{2}{*}{$\begin{array}{c}\text { Initial } \\
\mathrm{pH}\end{array}$} & \multirow{2}{*}{$\begin{array}{l}\text { Lowest } \\
\mathrm{pH}\end{array}$} & \multirow{2}{*}{$\begin{array}{c}\text { Highest } \\
\text { pH }\end{array}$} & \multicolumn{2}{|c|}{ AH test results } \\
\hline Group & Strain & & & & $\begin{array}{l}\text { Study of } \\
\text { Tully et al. }\end{array}$ & $\begin{array}{l}\text { This } \\
\text { study }\end{array}$ \\
\hline I-4 & $277 \mathrm{~F}$ & 7.7 & & & + & + \\
\hline I-5 & LB-12 & 7.3 & $7.2(5)^{b}$ & 7.2 & + & - \\
\hline I-6 & $\mathrm{M}^{2} 5^{\mathrm{T}}$ & 7.7 & $6.4(22)$ & 6.4 & + & - \\
\hline I-7 & N525 & 7.7 & & & + & + \\
\hline I-8 & $\mathrm{P} 40^{\mathrm{T}}$ & 7.7 & $7.5(6)$ & $\geq 8.4(30)$ & + & + \\
\hline VII & MQ-1 ${ }^{\mathrm{T}}$ & 7.7 & $6.2(4)$ & 6.2 & - & - \\
\hline VIII-3 & TAAS-1 & 7.7 & $6.8(2)$ & $\geq 8.4(30)$ & $\mathrm{ND}^{c}$ & + \\
\hline XIV & $\mathrm{EC}-1^{\mathrm{T}}$ & 7.7 & $\leq 6.0(3)$ & $6.2(19)$ & - & - \\
\hline XVIII & TAAS-2 & 7.7 & $\leq 6.0(4)$ & $\leq 6.0$ & ND & - \\
\hline $\mathrm{XX}$ & $\mathrm{LD}^{-1}{ }^{\mathrm{T}}$ & 6.4 & $\geq 7.4$ & $\geq 7.4(15)$ & + & + \\
\hline XXII & $\mathrm{CT}-1^{\mathrm{T}}$ & 7.7 & $\leq 6.0(11)$ & 6.4 (19) & - & - \\
\hline XXIII & TASS-1 & 7.7 & $\leq 6.0(8)$ & $\leq 6.0$ & ND & - \\
\hline XXV & $\mathrm{CUAS}^{-1}{ }^{\mathrm{T}}$ & 7.7 & $6.2(2)$ & 6.2 & - & - \\
\hline $\mathrm{UG}^{d}$ & BIUS-1 & 7.7 & $6.2(4)$ & 6.2 & ND & - \\
\hline UG & HYOS-1 & 7.7 & $6.4(3)$ & $8.3(30)$ & ND & + \\
\hline UG & PALS-1 & 7.7 & $6.8(2)$ & $8.2(30)$ & ND & + \\
\hline UG & PLHS-1 & 7.7 & $6.6(7)$ & $8.1(26)$ & ND & + \\
\hline UG & TABS-1 & 7.7 & $6.2(6)$ & 6.2 & ND & - \\
\hline UG & TABS-2 & 7.7 & $\leq 6.0(4)$ & $\leq 6.0$ & ND & - \\
\hline UG & TALS-2 & 7.7 & $6.2(7)$ & 6.2 & ND & - \\
\hline UG & TAUS-1 & 7.7 & $\leq 6.0(4)$ & $\leq 6.0$ & ND & - \\
\hline
\end{tabular}

${ }^{a}$ Data from reference 18 .

${ }^{b}$ The values in parentheses are the numbers of days after the culture was inoculated.

${ }^{c} \mathrm{ND}$, not determined.

${ }^{d} \mathrm{UG}$, ungrouped.

obtained for other AH-negative spiroplasmas, which lowered the $\mathrm{pH}$ to $\leq 6.0$ to 6.2 (Table 1). In trials in which the $\mathrm{M55}^{\mathrm{T}}$ culture $\mathrm{pH}$ was measured with an electrode, the $\mathrm{pH}$ values declined in all media tested as follows (the day on which the last motile form was observed microscopically is indicated in parentheses): in SFGA35 medium, from $\mathrm{pH} 7.0$ to 5.9 (day 56); in MTBSM medium, from $\mathrm{pH} 7.0$ to 5.6 (day 42); and in TWH medium, from $\mathrm{pH} 6.8$ to 6.3 (day 35). In samples collected at later times, all cells were nonmotile and presumably dead, although the $\mathrm{pH}$ continued to decline an additional 0.02 to 0.6 $\mathrm{pH}$ unit. When strain $\mathrm{M}^{\mathrm{T}} 5^{\mathrm{T}}$ was cultured at a low $\mathrm{pH}(\mathrm{pH} 6.0$ to 6.3 ), it multiplied and produced motile forms for up to 64 days in all three media, and the medium $\mathrm{pH}$ values declined slightly from 6.1 to 6.0 (SFGA35 medium), from 6.0 to 5.2 (MTBSM medium), and from 6.3 to 5.9 (TWH medium).

Group XX strain LD-1 ${ }^{\mathrm{T}}$ had previously been reported to be AH positive (18). This was confirmed in the current study (Table 1). In contrast to other groups, however, strain LD- ${ }^{\mathrm{T}}$ first raised (by $0.4 \mathrm{pH}$ unit) and then lowered (by $0.4 \mathrm{pH}$ unit) the $\mathrm{pH}$ in DBF medium and DBFG medium. In DBFA medium and DBFGA medium the $\mathrm{pH}$ increased to $\geq 7.4$; the $\mathrm{pH}$ did not decrease below a value of 7.4 for the duration of the test (30 days), at which time the cells were in the death phase. We suspect that the LD- ${ }^{\mathrm{T}}$ results were a consequence of peculiarities of the DCCM-based medium (which had an initial $\mathrm{pH}$ of 6.4) rather than the spiroplasma, for when arginineutilizing group IX strain $\mathrm{CN}-5^{\mathrm{T}}$ (18) was placed in DBFGA medium, it exhibited a similar $\mathrm{pH}$ reversal (the peak $\mathrm{pH}, \mathrm{pH}$ 7.0 , occurred at 5 days, and then the $\mathrm{pH}$ declined to $\leq 5.8$ at 13 days postinoculation).

Previous negative $\mathrm{AH}$ test results (18) were confirmed (Table 1) for the following previously grouped spiroplasma strains: MQ-1T (group VII), EC-1 ${ }^{\mathrm{T}}$ (group XIV), CT-1 ${ }^{\mathrm{T}}$ (group XXII), and CUAS-1 ${ }^{\mathrm{T}}$ (group XXV). In the current study (Ta- 


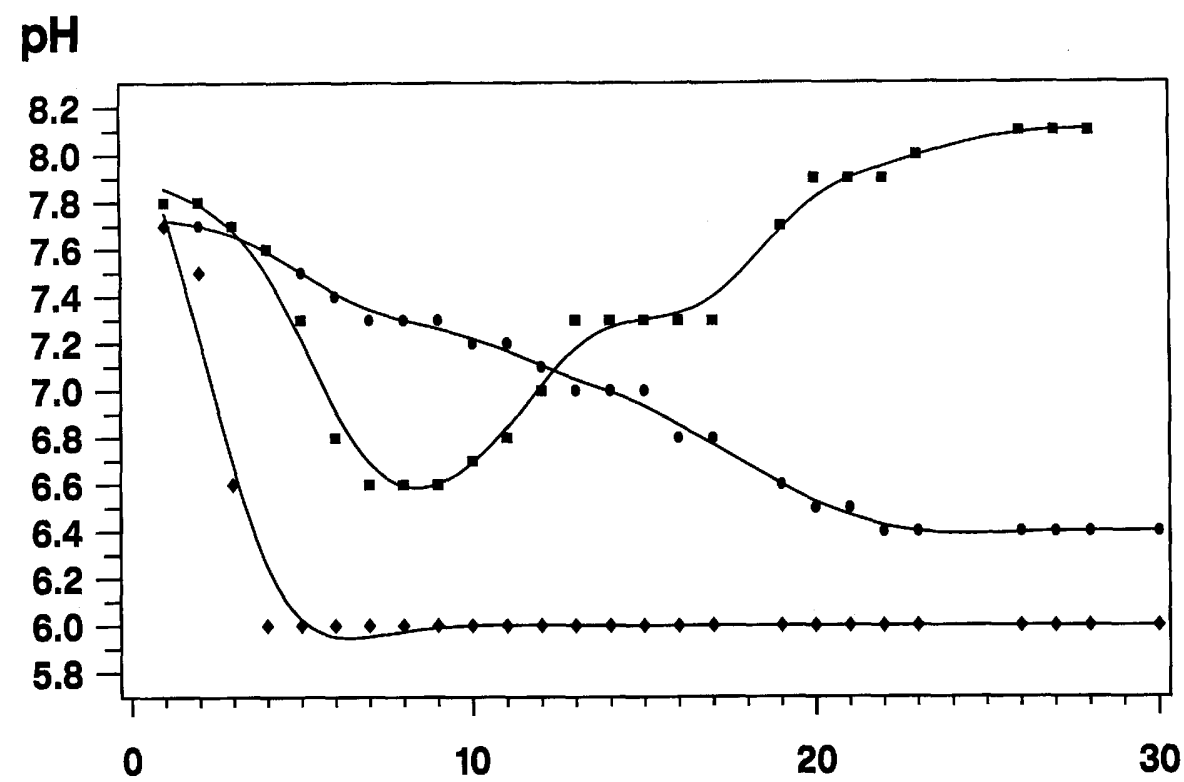

\section{Day}

FIG. 1. Growth of spiroplasmas in SF medium containing arginine (medium SFA). Symbols: $\bullet$ and $\bullet$ AH-negative strains TAUS-1 and M55 ${ }^{\mathrm{T}}$, respectively; arginine-positive strain PLHS-1.

ble 1), new strains placed in existing groups were $\mathrm{AH}$-positive group VIII-3 strain TAAS-1 and AH-negative group XVIII strain TAAS-2 and group XXIII strain TASS-1; these results are consistent with the results reported previously (18) for the strains that were representative of the groups.

Three new ungrouped strains (HYOS-1, PALS-1, and PLHS-1) were AH positive (Table 1). All other ungrouped strains (strains BIUS-1, TABS-2, and TALS-2, as well as serologically related strains TABS-1 and TAUS-1) were AH negative (data not shown).

With the exception of the strain $M 55^{\mathrm{T}}$ profile (Fig. 1), the AH-negative profiles in SFA medium were similar to the profile obtained for strain TAUS-1 (Fig. 1), in which $\mathrm{pH}$ values of $\leq 6.0$ to 6.2 were achieved within 2 to 11 days. For AH-positive strains (e.g., strain PLHS-1) (Fig. 1) the $\mathrm{pH}$ values of the media decreased as acid was produced during glucose metabolism and then increased as ammonia, which was produced during arginine metabolism, accumulated. Ammonia raised the $\mathrm{pH}$ level to 8.0 within 19 to 30 days, and the $\mathrm{pH}$ values sometimes reached $\geq 8.4$.

\section{DISCUSSION}

In the initial description of the genus Spiroplasma (11), the arginolytic ability of Spiroplasma citri was overlooked. The subsequent demonstration (15) that hydrolysis of this substrate by $S$. citri requires the presence of glucose and is delayed until latter parts of the growth cycle provided an explanation for the previous failure to detect the activity. Because we were unable to demonstrate $\mathrm{AH}$ by group I-5 and I- 6 spiroplasmas in media with and without glucose, we presume that the initial report of arginolytic activity by these two strains was in error and amend the previous conclusions.

In our studies, acid production in the presence of a high level of glucose $(0.5 \%$ glucose in SFGA medium) may have obscured ammonia production from arginine. An alternative ex- planation-that, as a consequence of repression of the arginine dihydrolase pathway by glucose fermentation, ammonia was not produced-seems less likely. Instead, evidence suggests that (i) arginine and glucose catabolic pathways are independent (11); (ii) arginine uptake is neutrogenic (i.e., it does not depend on a lowered $\mathrm{pH}$ [over a $\mathrm{pH}$ range of 5.0 to 8.0 ], as would result from glucose fermentation) (13); and (iii) the membrane protein for arginine-ornithine exchange is constitutively expressed in spiroplasmas (13) and other mollicutes (8). We suspect, therefore, that, despite being produced, ammonia did not accumulate to levels high enough to perceptively raise the $\mathrm{pH}$ (above $\mathrm{pH} 6.0$ ).

It should be emphasized that the AH test used in this study is a useful taxonomic tool but that this test does not elucidate the mechanism of arginine utilization or definitively establish the absence of $\mathrm{AH}$. We did not analyze spiroplasmas for enzymes of the arginine dihydrolase pathway or measure ammonia production or arginine catabolism. Perhaps some AH-negative spiroplasmas produced low amounts of ammonia. If so, this could explain the weak acidification of media by strains LB-12 and M55 ${ }^{\mathrm{T}}$, a metabolic capability that, in nature, could function to prolong maintenance of these spiroplasmas on or in their hosts.

The $\mathrm{AH}$ test nevertheless continues to be an important diagnostic tool for spiroplasma species and should continue to be of substantial use in taxonomy, particularly-because the ability to use arginine occurs in various spiroplasma lineages (19) and appears to be rather evolutionarily labile-for classifying organisms at lower taxonomic levels.

A standardized protocol is not currently possible because of the difficulty of growing fastidious spiroplasmas in SF-based media. However, for most spiroplasmas, SF-based media that contain penicillin and are sterilized by filtration are suitable. When $0.02 \mu \mathrm{g}$ of phenol red per $\mathrm{ml}$ is used as the indicator, arginine metabolism and glucose metabolism can be determined by adding $0.42 \% \mathrm{~L}$-arginine- $\mathrm{HCl}$ (purple color change) 
and $0.5 \%$ glucose (yellow color change), respectively. The protocol of Barile (2) and Aluotto et al. (1), with the following modifications, is recommended: (i) the organism should be adapted from M1D medium to FBSI medium and passaged three or four times; (ii) portions of a high-dilution log-phase FBSI medium culture should be inoculated into the SF-based test media (SF, SFG, SFA, and SFGA media) adjusted to $\mathrm{pH}$ 7.7; and (iii) the preparations should be incubated and observed daily for color changes by using SFGA medium titrated at gradations of $0.1 \mathrm{pH}$ unit ( $\mathrm{pH} 5.8$ to 7.4) as the color standard set. Arginine-positive organisms are organisms that raise the $\mathrm{pH}$ of SFA medium (the $\mathrm{pH}$ usually decreases for 2 to 7 days to values of 6.4 to 7.5 before it increases to values of $>8.0$ at 26 to 30 days postinoculation).

\section{REFERENCES}

1. Aluotto, B. B., R. G. Wittler, C. O. Williams, and J. E. Faber, 1970. Standardized bacteriologic techniques for characterization of Mycoplasma species. Int. J. Syst. Bacteriol. 20:35-58.

2. Barile, M. 1983. Arginine hydrolysis, p. 345-349. In S. Razin and J. G. Tully (ed.), Methods in mycoplasmology, vol. 1. Academic Press, Inc., New York.

3. Barile, M. F., R. T. Schmike, and D. B. Riggs. 1966. Presence of arginine dihydrolase pathway in Mycoplasma. J. Bacteriol. 91:189-192.

4. Hackett, K. J., and D. E. Lynn. 1995. Insect cell culture approaches in cultivating spiroplasmas, p. 55-64. In S. Razin and J. G. Tully (ed.), Molecular and diagnostic procedures in mycoplasmology, vol. 1. Academic Press, Inc., San Diego, Calif.

5. Hackett, K. J., and R. F. Whitcomb. 1995. Cultivation of spiroplasmas in undefined and defined media, p. 41-54. In S. Razin and J. G. Tully (ed.), Molecular and diagnostic procedures in mycoplasmology, vol. 1. Academic Press, Inc., San Diego, Calif.

6. Hackett, K. J., R. F. Whitcomb, T. B. Clark, R. B. Henegar, D. E. Lynn, A. G. Wagner, J. G. Tully, G. E. Gasparich, D. L. Rose, P. Carle, J. M. Bové, M. Konai, E. A. Clark, J. R. Adams, and D. L. Williamson. 1996. Spiroplasma leptinotarsae sp. nov., a new species from the Colorado potato beetle, Leptinotarsa decemlineata (Coleoptera: Chrysomelidae). Int. J. Syst. Bacteriol. 46:906-911.

7. Miles, R. J. 1992. Catabolism in Mollicutes. J. Gen. Microbiol. 138:17731783 .

8. Olson, L. D., C. A. Renshaw, S. Rottem, and J. H. Boal. 1993. Arginine utilization by Mycoplasma fermentans is not regulated by glucose metabolism: a 13C-NMR study. FEMS Microbiol. Lett. 108:47-52.

9. Patterson-Curtis, S. I., and E. A. Johnson. 1992. Roles of arginine in growth of Clostridium botulinum Okra B. Appl. Environ. Microbiol. 58:2334-2337.

10. Reinsch, C. H. 1967. Smoothing by spline functions. Numerische Math. 10:177-183.

11. Saglio, P., M. L'Hospital, D. Laflèche, G. Dupont, J. M. Bové, J. G. Tully, and E. A. Freundt. 1973. Spiroplasma citri gen. and sp. n.: a mycoplasma-like organism associated with "stubborn" disease of citrus. Int. J. Syst. Bacteriol. 23:191-204.

12. SAS Institute, Inc. 1990. SAS graph software, version 6, 1st ed., vol. 1. SAS Institute, Inc., Cary, N. C.

13. Shirazi, I., M. Tarshis, and S. Rottem. 1995. An arginine-ornithine exchange system in Spiroplasma melliferum cells. Microbiology 141:2323-2328.

14. Stevens, C., R. M. Cody, and R. T. Gudauskas. 1983. Effects of arginine on growth and in prolonging longevity of corn stunt spiroplasma in vitro. J. Gen. Appl. Microbiol. 29:1-7.

15. Townsend, R. 1976. Arginine metabolism by Spiroplasma citri. J. Gen. Microbiol. 94:417-420.

16. Tully, J. G., J. M. Bové, F. Laigret, and R. F. Whitcomb. 1993. Revised taxonomy of the class Mollicutes: proposed elevation of a major cluster of arthropod-associated mollicutes to ordinal rank (Entomoplasmatales ord nov.), with provision for familial rank to separate species with nonhelical morphology (Entomoplasmataceae) from helical species (Spiroplasmataceae), and emended descriptions of the order Mycoplasmatales, family $M y$ coplasmataceae. Int. J. Syst. Bacteriol. 43:378-385.

17. Tully, J. G., and S. Razin (ed.). 1995. Molecular and diagnostic procedures in mycoplasmology, vol. 2, p. 460-462. Academic Press, Inc., San Diego, Calif.

18. Tully, J. G., D. L. Rose, E. Clark, P. Carle, J. M. Bové, R. B. Henegar, R. F. Whitcomb, D. E. Colflesh, and D. L. Williamson. 1987. Revised group classification of the genus Spiroplasma (class Mollicutes), with proposed new groups XII to XXIII. Int. J. Syst. Bacteriol. 37:357-364.

19. Weishurg, W. G., J. G. Tully, D. L. Rose, J. P. Petzel, H. Oyaizu, D. Yang, L. Mandelco, J. Sechrest, T. G. Lawrence, J. Van Etten, J. Maniloff, and C. R. Woese. 1989. A phylogenetic analysis of the mycoplasmas: basis for their classification. J. Bacteriol. 171:6455-6467.

20. Whitcomb, R. F., and K. J. Hackett. 1995. Identification of mollicutes from insects, p. 313-322. In J. G. Tully and S. Razin (ed.), Molecular and diagnostic procedures in mycoplasmology, vol. 2. Academic Press, Inc., San Diego, Calif.

21. Williamson, D. L. 1989. The genus Spiroplasma, p. 71-111. In R. F. Whitcomb and J. G. Tully (ed.), The mycoplasmas, vol. 5. Academic Press, Inc., New York. 